\title{
On the construction of wear maps for Y-TZP dental ceramics in aqueous environments: pH, exposure time and impact angle effects
}

\author{
M. M. Stack ${ }^{1^{*}}$, Ting-Fu Hong ${ }^{2}$, Hsin-Chun Wang ${ }^{3}$, Moo-Chin Wang ${ }^{4}$, Wang-Long \\ $\mathbf{L i}^{5}$ \\ 1 Department of Mechanical Engineering, University of Strathclyde, James Weir \\ Building, 75 Montrose Street Glasgow G1 1XJ, Scotland, UK
}

${ }^{2}$ Department of Materials Engineering, National Pingtung University of Science and Technology, 1, Hseuhfu Road, Neipu, Pingtung 91201, TAIWAN

${ }^{3}$ Institute of Nanotechnology and Microsystems Engineering, National Cheng Kung University, No.1 University Road, Tainan City 701, TAIWAN

${ }^{4}$ Faculty of Fragrance and Cosmetics, Kaohsiung Medical University, 100, Shihchuan 1st Road, Kaohsiung, 80728, TAIWAN

${ }^{5}$ Institute of Nanotechnology and Microsystems Engineering

National Cheng Kung University

No.1, University Road, Tainan, 701, TAIWAN

http://science.nchc.org.tw; http://myweb.ncku.edu.tw/ wlli

Tel:+886-6-2757575\#31396; Fax:+886-6-2745885; Mobil:+886-920682641 


\begin{abstract}
The purpose of this study was to test in-vitro the micro and nano tribological properties of Y-TZP (Yttria Tetragonal Zirconia Polycrystalline) material used in the construction of artificial teeth or dental crowns. The results showed that the effect of $\mathrm{pH}$ was to accelerate the wear rate of the Y-TZP material. However, this phenomenon was more apparent in acid than in alkaline environments. Wear maps were constructed based on the results identifying wastage regimes for the material in the erosion-corrosion conditions at a range of impact angles. In addition, a wear map was generated based on the transition from tetragonal to monoclininc phase for the material and the transition to high wear at various $\mathrm{pHs}$ and exposure times.
\end{abstract}

Key words: Wear Map, Dental Materials, Y-TZP, Solid Particle Impact, Aqueous Slurry, pH, Impact Angle and Exposure Time 


\section{Introduction}

Tribology and Tribo-Corrosion in Dentistry is becoming of increasing international research interest as it is widely observed that human teeth are exposed to several tribological processes inside the oral cavity [1]. These effects can be characterized into five types: attrition (the impact between enamel surfaces), abrasion (wear induced by friction with food or toothpaste particles), abfraction (loss of dental material induced by cracks in tooth enamel), corrosion (chemical reactions inside the oral cavity) and erosion (slurries containing particles that impact enamel surfaces) [1]. Due to these effects, human teeth will always be susceptible to both mechanical and chemical degradation.

Corrosion is also a very significant issue within the oral cavity, as the $\mathrm{pH}$ value of human saliva is approximately 6.2 [2]. Furthermore, it can be significantly decreased to 2 on consumption of acidic food or drinks. Sometimes, it may be mixed with backflow hydrochloric acid present in gastric juices, causing $\mathrm{pH}$ values to be reduced to 1.2 [2]. On the other hand, food such as fruit or kelp can induce an increase in $\mathrm{pH}$ value up to 10 [2].

Erosion effects are caused by particles within a solution impacting material surfaces. Erosion occurs daily in the human oral cavity; each time people drink beverages or masticate food, small particles will be mixed with liquid, which erode teeth surfaces.

Today, due to aesthetic and anti-allergy reasons, ceramic artificial teeth or dental crowns are used to restore or replace damaged nature teeth [3]. The principle material for these dental restoration products is Y-TZP (Yittria-Tetragonal Zirconia 
Polycrystalline). Y-TZP can be sintered to the color natural teeth. Additionally, due to its unique ductility among other ceramics, Y-TZP material is found to be wear resistant under repeated mastication [3]. Y-TZP material is also significantly bio-compatible, which can reduce the chance of allergy in patients [4]. Hence, its suitability as a ceramic material for creating artificial teeth.

(In Dentistry, the term erosion is used to indicate dissolution but in Mechanical Engineering it is used in a different sense to indicate mechanical wear. Here, corrosion is the term generally describing the processes of acid dissolution and formation of passive films. Erosion-corrosion is the process where impact by solid particles can interact with chemical degradation of the surface. For the purpose of this paper, clarification of these terms will be made throughout the text in order to attempt to unify the nomenclature to this interdisciplinary community).

When brittle materials such as ceramics are subjected to erosion, radial and lateral cracks will form on the surface [5-9]. If corrosion interacts with erosion, there are many possible effects, i.e. additive, synergistic or antagonistic effects [10]. In such cases, the wear rate may be significantly greater or less than the pure erosion or corrosion effects [11-16].

As candidate replacement dental materials, ceramic materials have disadvantages due to their brittle nature. Once cracks form inside the materials, propagation of damage cannot be suppressed [17-19]. However, it has been found that Y-TZP possesses enhanced ductility compared to many conventional ceramics as cracks propagation tends to less in the latter case [20]. These properties are due to the Martensitic phase transformation of Y-TZP material from tetragonal to monoclinic. The phase transformation can induce a $3 \%-5 \%$ change in volume that effectively suppresses 
crack propagation [20]. This example of phase transformation of Y-TZP material can be induced not only by erosion and corrosion, but also by various physical, thermal and chemical effects $[21,22]$.

In this paper, the effects of erosion on Y-TZP material were assessed at various impact angles, $\mathrm{pH}$ values and exposure times. Dental materials experience degradation at various impact angles in the oral environment by food particles and hence is the reason why the effects of this parameter were investigated. Such an analysis facilitated the construction of a new wear mapping approach to classifying wear rates and mechanisms of dental materials, for the various mechanical and chemical interactions taking place in the oral environment. 


\section{Experimental Details}

\subsection{Apparatus}

The erosion behaviour of the Y-TZP material was evaluated using an Impinging Jet Erosion Apparatus (described elsewhere; [23, 24]) based in the Department of Mechanical Engineering, University of Strathclyde, Scotland. The schematic diagram of the experimental rig is given in fig. 1. The impact angle varies between $15^{\circ}$ and $90^{\circ}$ and the velocity was set at $4.39 \mathrm{~ms}^{-1}$. The slurry was composed of 12.29 wt. $\% \mathrm{SiO}_{2}$ particles (12.29 wt. \% particles to 87.71 wt. \% solution) with diameters ranging from 300 to $600 \mu \mathrm{m}$ in acid, alkaline or neutral solutions. The acid solution of $\mathrm{pH} 2.0,3.5$ and 5.0 were mixed with $\mathrm{HCl}$ in deionised water. For alkaline solutions, the $\mathrm{pH} 8.5$ and 9.5 were mixed with $\mathrm{NaOH}$ in deionised water. A nanoindenter was used to measure mechanical resistance, and X-Ray Diffraction (XRD) was used to assess the crystal structure of the samples. The other experimental parameters are shown in Table.1.

\subsection{Preparation of Specimens}

Circular-disc specimens of 9-mm diameter and 2.25-mm thickness were prepared with 3 mole \% yittria doped zirconia. The Zircar ${ }^{\circledR} 3$ mole\% yttria stablized zirconia powder was sintered at $1500^{\circ} \mathrm{C}$ for $4 \mathrm{hr}$. All specimen surfaces were polished sequentially with $6,3,1$, and $0.3-\mu \mathrm{m}$ diamond paste. Between the polishing steps, the specimens were ultrasonically cleaned in de-ionized water to remove any polishing debris.

\subsection{Analysis of Mass Loss}


Each specimen was eroded by Impinging Jet Erosion Apparatus for $20 \mathrm{~h}$ in different $\mathrm{pH}$ conditions. Each test results represents the mean of results for exposure of two different specimens. The specimens were weighed every hour (once per half hour for the first two hours). Before weighing, deionised water was used to clean the specimens. Warm air at $50^{\circ} \mathrm{C}$ was used to dry the specimens for $20 \mathrm{~min}$.

\subsection{Nanoindentation measurements}

The indentation tests were carried out with a MTS ${ }^{\circledR}$ G200 Nanoindenter accompanied by Berkovich indenter. An indentation depth of $300 \mathrm{~nm}$ was used. The radius of the indenter tip was 40nm. The Young's modulus and hardness values were calculated by the mean values of 200 to $300 \mathrm{~nm}$ according to the results of CSM (Continuous Stiffness Measurment). Each sample was subjected to 20 indentation tests.

\section{Results}

X-ray diffraction results of the original Y-TZP specimens are shown in Fig. 2. The tetragonal structure of zirconia was evident in the pre-exposed specimen. After the specimens were exposed to the erosion tests at various $\mathrm{pH}$ values, the intensity of the tetragonal zirconia appeared to decrease significantly, and there was evidence of monoclinic zirconia formed on the surface (Fig. 3-5). Table 2 shows the nanoindenter and Vickers indenter indentation results of the specimens before and after erosion tests. The results showed that the original sample presented the highest mechanical resistance. 
The hardness values of all the specimens after erosion tests appeared to be reduced when compared to their respective original specimens. SEM analysis of the Y-TZP specimens before exposure, Fig. 6- Fig.12, showed the surface morphology and the cross-section of the specimens after the erosion-corrosion tests. The images indicate that before the tests the particles were packed tightly; following exposure, the initial larger grains appeared to have fractured. The results of cumulative mass loss per unit area in different erosion-corrosion conditions are shown in Fig. 13. Table 3, displaying total mass loss after $20 \mathrm{~h}$ of erosion-corrosion tests, shows that the highest wastage occurs in erosion in the acidic slurry and the lowest in the neutral slurry. For various erosion conditions, the wear rate became constant after a specific erosion duration. Sudden increments of erosion in the period between 0.5 to $2 \mathrm{hr}$ were observed for the test results.

Fig. 14 to 17 show the cumulative mass loss curve at various impact angles. At shallow impact angles, the wear rate was almost similar to that at normal impact i.e. at 90 degrees. The main difference between the results at both impact angles was the critical time to the transition to high mass loss rates. 


\section{Discussion}

\subsection{Morphological changes in Y-TZP material following erosion-corrosion exposure}

The martensitic phase transformation of tetragonal zirconia is well known to be significant in Y-TZP material. Following sintering at $1500 \square$ for $4 \mathrm{hr}$, Fig. 2, only tetragonal zirconia can be identified in the Y-TZP specimen. However, after the specimens were subject to impact by solid particles for a specific period, there is evidence of monoclinic zirconia formation on the surface of the specimens. However, the monoclinic formation times are slightly different for each erosion conditions. For alkaline and neutral conditions, $1 \mathrm{hr}$ erosion time is required for monoclinic formation, whereas for acid conditions, only $0.5 \mathrm{hr}$ is required.

Since the main peak of tetragonal zirconia shifts after erosion tests in various $\mathrm{pH}$ conditions, some residual stresses exists in the specimen surface. Thus, cracks will form on the specimen surface. Although Y-TZP material is often termed as "Ceramic Steel" due to its high ductile strength relative to other ceramic materials, this material displays evidence of brittle mechanical behaviour [10]. Because the ductile property of Y-TZP is due to the phase transformation strengthening mechanism and not the inherent nature of this material, when this material is subjected to impact of erosive particles, cracks still can form within the material. As a result, the grains in the material will be fractured, and cracks will propagate below the surface. Based on Fig. 8, the cross-section image of the specimen after erosion tests, this layer of propagated cracks is approximately $2 \sim 3 \mu \mathrm{m}$ in thickness.

While the specimens were being subjected to erosion tests, various defects are likely 
to have formed on the boundaries of different grains that increase the contact surface between the specimens and their respective erosive solution, causing the corrosive wastage to become more severe. As a result, the mass loss of these specimens increases significantly as the surface area for corrosion will increase.

Although the results of nanoindentation show that the Young's modulus and hardness values decrease after the erosion tests were performed (table 2) no obvious differences exists between each experimental condition. According to XRD analysis, the formation of monoclinic zirconia should lead to reduction of mechanical strength. However, this reduction of mechanical strength cannot be observed in the Vickers hardness measurement. This is possibly due to the fact that the measuring scale of the Vickers indenter is much larger than nanoindenter and the transformation layer of the Y-TZP material. Therefore, hardness measurements using the Vickers indenter will not only be influenced by the hardness of the transformation layer of the Y-TZP material, but also the hardness of the tetragonal zirconia layer underneath. As a result, the hardness from Vickers indenter measurement will mix results from both the tetragonal and monoclinic zirconia, rendering mechanical strength readings through the Vickers indenter unreliable.

\subsection{Comparison between pH effects for the Y-TZP}

Fig. 13 indicates that all the mass loss curves can be divided into two groups - the first delineated by the curves obtained at $\mathrm{pH} 2.0$ and 3.5, and the second composed of the remaining curves at higher $\mathrm{pHs}$. Although the $\mathrm{pH} 5.0$ is also acidic, it belongs to the second group; thus, it can be concluded that although the total wastage at $\mathrm{pH} 5.0$ is 
much larger than other neutral or alkaline erosion conditions, there are no obvious differences between the curves. Only when the $\mathrm{pH}$ value is less than 3.5 is the wastage of Y-TZP material accelerated and dramatically increased confirming that below this critical $\mathrm{pH}$ value, a transition to a different mechanism of erosion-corrosion behaviour takes place.

Equations 1 and 2 are the possible reactions between hydrochloride acid, sodium hydroxide and tetragonal zirconia [5].

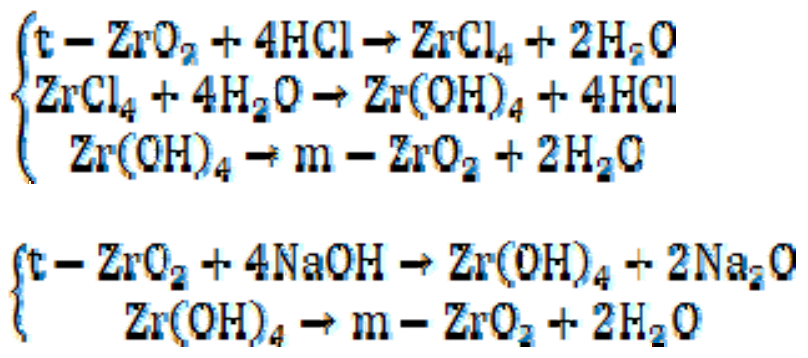

Therefore, corrosive effects from both acid and alkaline solutions may induce the phase transformation of partially stablized zirconia material from tetragonal to monoclinic. In this study, pure corrosion tests performed with acid or alkaline solutions without erosive particles are also obtained. The results show that after $20 \mathrm{~h}$ tests, only mass losses of $0.007 \mathrm{kgm}^{-2}$ and $0.006 \mathrm{kgm}^{-2}$ at $\mathrm{pH} 2.0$ and 9.5, were recorded respectively. Compared to the result $0.286 \mathrm{kgm}^{-2}$ of erosion in neutral solution, it is found that the effect of erosion is more significant than corrosion. As a result, when these two effects exist simultaneously, the erosion (by solid particles) effect dominates.

The sum of neutral erosion and corrosion in a solution of $\mathrm{pH} 2.0$ is $0.290 \mathrm{kgm}^{-2}$. This value is much smaller than the wastage of acid erosion ( $\mathrm{pH} 2.0$ with erosive particles). When metallic materials are subjected to erosion-corrosion effects in acidic conditions, the wastage may be much greater than by the separate processes of erosion or corrosion 
[24-26]. The results from the above study indicate that with the formation of craters or cracks on the surface, more material is exposed to corrosive slurry, causing significant increase in corrosion-induced phase transformation. Based on the findings from nanoindentation on the specimens before and after the erosion-corrosion tests, the mechanical hardness of the specimens decreases after the tests due to the presence of monoclinic zirconia on the specimen surface. As a result, this may indicate that erosion effects accelerate the corrosion-induced phase transformation, and mechanical resistance of the specimens decreases while the wastage of the specimens significantly increases. Hence, the erosion-corrosion interactions are complex and involve phase transformation, reduction in mechanical strength, increase in flaws and fracture, and increase in surface area for corrosion. All of these contribute to an enhancement in wastage at low $\mathrm{pH}$ values in the erosion-corrosion environment.

\subsection{Construction of wear maps}

Through identification of wastage levels as $30 \%$ and $80 \%$ of the maximum accumulated mass loss, three wear levels of the Y-TZP specimens (Low, Medium and High) were defined. The results plotted on a two dimensional representation are shown in Fig. 18 as a wear map. The following equations are the definitions of the levels: (the unit of wear is $\mathrm{kgm}^{-2}$

Wear $\leq \leqq 0.100 \rightarrow$ Low

$0.0100<<$ Wear $<0.270 \leq \rightarrow$ Medium

Wear $>\geqq 0.270 \rightarrow$ High 
Fig.18 shows that the transition to the various "Medium" and "High" regimes occurs at longer times in neutral solution than for the other conditions. In other words, corrosion significantly accelerates the wear of the specimens. Comparing the acidic and alkaline solutions, the first boundary (the boundary between "Low" and "Medium") shows that the time required for alkaline erosion to reach the "Medium" region is longer than that required for acid erosion. According to the second boundary (the boundary between "Medium" and "High"), this phenomenon is exaggerated; the erosion-corrosion wear in acid is much more severe than in alkaline solutions. Thus, Y-TZP material has less erosion-corrosion resistance to acid erosion conditions than in alkali solutions.

Based on the maximum wear rates in the initial erosion (by solid particles) period and the minima of the period in which the dramatic mass loss appeared, three levels of wear rate of the Y-TZP specimens (Low, Medium and High) are also defined. Fig.19 shows the wear rate map of the Y-TZP material. The definitions of different levels are shown below: (the unit of wear rate is $\mathrm{kgm}^{-2} \mathrm{~h}^{-1}$

Wear rate $\leqq \leq 0.010 \rightarrow$ Low

$0.0010<$ Wear rate $\leq<0.040 \rightarrow$ Medium

Wear rate $>0.040 \rightarrow$ High

As a result of the excellent mechanical resistance of tetragonal zirconia, Fig. 19 shows that when the specimens were initially eroded by the erosive slurries, the wear rates are "Low" level in all exposure conditions. After exposure for longer times, the wear rates acquire steady values in the medium range, they initially enter the high range; this is due to the sudden phase transformation in the materials. When the monoclinic zirconia suddenly becomes exposed on the surface, the mechanical properties of the 
specimen decrease in a very short period; during this duration, if the specimen is subjected to the impact of erosive particles, the surface material is likely to degrade very significantly. Therefore, a very significant sudden increase in mass loss can be observed.

Due to the propagation of the cracks formed on the surface, it is likely that the zirconia materials beneath the surface will transform into monoclinic zirconia before the removal of the surface material. After the collapse of the surface material, the exposed material remains in the monoclinic zirconia, ensuring the wear rate of the material remain in the "Medium" region after specific erosion times.

The results also show that the acidic erosive conditions affect the martensitic phase transformation of Y-TZP material much more noticeably than other conditions, and that they induce the Y-TZP material to pass through the transitional high wear rate region rapidly before remaining in the steady medium wear rate region. On the other hand, the result of alkaline erosion shows that the locations at these two boundaries are almost the same as the locations under neutral conditions. Therefore, the alkaline erosive condition will not affect the Y-TZP materials seriously, and its erosion-corrosion results are similar to those under neutral erosive conditions.

For impact at oblique angles, Fig. 21-23, the wear maps show very different transition boundaries to those observed at 90 degrees, Fig. 19-20. Clearly, this has to be due to be due to the difference of the resistance of the material at such impact angles compared to that observed for normal impacts, Fig. 19-20. In general, the transition exposure time from low to medium high wear rate is reduced with increasing impact angles. This is consistent with results on the solid particle erosion of ceramics where erosion rates are higher than those at lower impact angles due to higher crack propagation rates at normal impacts [6]. 
Based on the above analysis, a phase transformation wear map can be generated for erosion-corrosion of this material, based on the tetragonal to monoclinic transformation, Fig. 24. Here, it can be seen over this parameter space, the monoclinic phase transition is favoured at low pHs. This indicates that impact of solid particles in aqueous conditions accelerates this transition, with the transition occurring at shorter exposure times at low pHs. Given that the tests carried out were accelerated in terms of the mechanical interaction i.e. the use of large particles and concentrations, it is likely that in oral environments in less aggressive conditions, this transition time will be longer. However, the accelerated transition is still likely to occur at the lower $\mathrm{pH}$ values, as indicated by this phase transformation wear map, Fig. 24.

Further work will be to assess whether such patterns in erosion-corrosion behaviour are observed for other dental replacement materials and how such maps can be used to optimize the performance of a wide range of alternative materials for exposure to such conditions.

\section{Conclusions}

a. Wear by the impact of solid particles and corrosion by exposure to various $\mathrm{pH}$ values results in a phase transformation of a Y-TZP material. The synergistic effect of wear and corrosion effects both induce a phase transformation of Y-TZP material, from the tetragonal to monoclinic phase, resulting in a significant increase in wear rate which is attributed to the lower wear resistance of the monoclinic phase in such conditions. 
b. The Y-TZP material undergoes significantly more wastage in acidic compared to alkaline solutions and particularly below $\mathrm{pH}$ values of 3.5. When the Y-TZP material is subjected to erosion-corrosion effects, initially the degree of zirconia formed on the surface is low; only after a significant exposure time to particle impacts is the phenomenon easily observed.

c. Wear maps can be constructed identifying wastage regimes for the material studied in the various $\mathrm{pH}$ and exposure times studied. Such maps can be used to identify transitions in wastage levels and mechanistic changes in the material following erosion-corrosion as identified by XRD and nanomechanical analysis .

\section{Acknowledgements}

This investigation was supported in part by Research Grant from the National Science Council (NSC), Taiwan, contract no.: NSC-97-2221-E-006- 223-. The authors would like extend thanks to the Ministry of Education and NSC Taiwan for sponsorship of Prof M M Stack's visits to Taiwan and the Royal Society of Edinburgh and NSC Taiwan for Professor Li's visit to University of Strathclyde, Glasgow, UK.

\section{References}

[1] Grippo JO, Simring M, Schreiner S. Attrition, abrasion, corrosion and abfraction revisited: A new perspective on tooth surface lesions. J Am Dent Assoc, 2004; 135(8):1109-18.

[2] Moazzez R, Smith BGN, Bartlett DW. Oral pH and drinking habit during ingestion of a carbonated drink in a group of adolescents with dental erosion. Am J Dent, 2004; 28(6):395-7. 
[3] Liu KS, Lin SC, Li SL, Cheng HC, Yeh CW. Engineering Material Science, Taipei: Chuan Hwa Book Coorperation; 1991. p. 313.

[4] Suzuki O, Suda A, Sato T, Takagi M, Osanai T. Biocompatibility of zirconia dispersed hydroxyapatite ceramics. J Jpn Ortho Assoc, 1990; 64(4):249-58.

[5] Fang Q, Sidky PS, Hocking MG. The effect of corrosion and erosion on ceramic materials. Corros Sci, 1996; 39(3):511-27.

[6] Srinivasan S, Scattergood RO. Effect of erodent hardness on erosion of brittle materials. Wear, 1988; 128(2):139-52.

[7] Ritter JE. Spherical particle impact damage. Key Eng Mater, 1992; 71:93-106.

[8] Rowcliffe DJ. Quasi-static indentation of ceramics. Key Eng Mater, 1992; $71: 1-22$.

[9] Fang Q, Xu H, Sidky PS, Hocking MG. Erosion of ceramic materials by a sand/water slurry jet. Wear, 1999; 224(2):183-93.

[10] Stack MM, Pumgwiwat N. Particulate erosion-corrosion of Al in aqueous conditions: some perspectives on $\mathrm{pH}$ effects on the erosion-corrosion map. Tribol Int, 2002; 35(10):651-60.

[11] Batchelor AW, Stachowiak GW. Predicting synergism between corrosion and abrasive wear. Wear, 1988; 123(3):281-91.

[12] Postlethwaite J, Tinker EB, Hawrylak MW. Erosion-corrosion in slurry pipelines. Corrosion, 1974; 30(8):285-90.

[13] Madsen BW. Measurement of erosion-corrosion synergism with a slurry wear test apparatus. Wear, 1988; 123(2):127-42. 
[14] Stack MM, Bridging the gap between tribology and corrosion: from wear maps to Pourbaix diagrams, Int Mater Rev, 2005; 50(1):1-17.

[15] Stack MM, Jana BD, Modelling particulate erosion-corrosion in aqueous slurries: some views on the construction of erosion-corrosion maps for a range of pure metals, Wear, 2004; 256(9-10):986-1004.

[16] Stack MM, Antonov MM, Hussainova I, Some views on the erosion-corrosion response of bulk chromium carbide based cermets, J Phys D: Appl Phys, 2006; 39:3165-3174.

[17] Srinivasan S, Scattergood RO, Effect of erodent hardness on erosion of brittle materials, Wear, 1988; 128(2):139-152.

[18] Ritter JE, Spherical particle impact damage, Key Eng Mater, 1992; 71: 93-106.

[19] Rowcliffe DJ, Quasi-static indentation of ceramics, Key Eng Mater, 1992; 71: $1-22$.

[20] Stevens R, An introduction to zirconia. London: Magnesium Electron Press; 1986.

[21] Lay L. Corrosion resistance of technical ceramics. London: HMSO Publish Centre; 1991. p. 56-58.

[22] McCauley RA. Corrosion of ceramics. New York: Marcel Dekker Press; 1995. p. 145.

[23] Zu JB, Hutchings IM, Burstein GT. Design of a slurry erosion test rig. Wear, 1990; 140(2):331-44. 
[24] Stack MM, Pungwiwat N. Erosion-corrosion mapping of $\mathrm{Fe}$ in aqueous slurries: a new rationale for defining the erosion-corrosion interaction. Wear, 2004; 256(5):565-576.

[25] Stack MM, Abdelrahman SM and Jana BD, A new methodology for modelling erosion-corrosion regimes of real surfaces: gliding down the galvanic series for a range of metal corrosion systems" Wear, 2010; 268 (3-4):533-542.

[26] Purandare,Y.P. Ehiasarian A.P., Stack MM and Hovsepian P.Eh,CrN/NbN Coatings deposited by HIPMS: A preliminary study of erosion-corrosion performance" Surface and Coatings Technology 2010; 204 (8): 1158-1162, 2010.

[27] Stack M.M. and Abdulrahman, Mapping erosion-corrosion of carbon steel in oil exploration conditions: Some new approaches to characterizing mechanisms and synergies", Tribology International, 2010, 43 (7)1268-1277. 


\section{Captions for Tables}

Table 1 - Experimental Parameters

Table 2 - Mechanical properties of the Specimens

Table 3 - Total Mass Loss of the specimens after $20 \mathrm{~h}$ exposure 


\section{Tables}

\section{Table 1 - Experimental Parameters}

\section{Parameters}

$\boldsymbol{A}_{\boldsymbol{z}}$ (Surface area of the specimen)

$c$ (Sand concentration of the slurry)

$\boldsymbol{d}$ (Diameter of the driving nozzle)

D (Diameter of the exit nozzle)

$\boldsymbol{D}_{2}$ (Diameter of the suction tube)

$\boldsymbol{d}_{z}$ (Diameter of the specimen)

$\boldsymbol{L}_{1}$ (Length of the ejector)

$\boldsymbol{L}_{2}$ (Length of the exit nozzle)

$v$ (Velocity of the slurry)

$V_{f}$ (Volume fraction)

$\boldsymbol{x}$ (The distance between the end of driving nozzle and centerline of the suction tube)

$\boldsymbol{x}_{\boldsymbol{z}}$ (The distance between the end of exit nozzle and out surface of the specimen)

$\boldsymbol{\rho}_{\boldsymbol{s}}$ (Density of the sand)

$\boldsymbol{\rho}_{z \boldsymbol{o}}$ (Origin density of the specimen)
Experimental values

$6.360 \times 10^{-5} \mathrm{~m}^{2}$

12.29 wt. $\%$

$0.003 \mathrm{~m}$

$0.005 \mathrm{~m}$

$0.0063 \mathrm{~m}$

$0.009 \mathrm{~m}$

$0.003 \mathrm{~m}$

$0.0035 \mathrm{~m}$

$4.39 \mathrm{~m} \mathrm{~s}^{-1}$

9.58 vol.\%

$-0.0079 \mathrm{~m}$

$0.005 \mathrm{~m}$

$2230.4 \mathrm{~kg} \mathrm{~m}^{-3}$

$7631.2 \mathrm{~kg} \mathrm{~m}^{-3}$

(Section 2.1.) 


\section{Table 2 - Mechanical properties of the Specimens}

\begin{tabular}{|c|c|c|c|c|c|c|}
\hline $\begin{array}{l}\text { Experimental } \\
\text { conditions }\end{array}$ & $\begin{array}{l}\text { Young's } \\
\text { Modulus } \\
\text { (GPa) }\end{array}$ & S.D. & $\begin{array}{l}\text { Hardness } \\
\text { (GPa) }\end{array}$ & S.D. & $\begin{array}{l}\text { Vickers } \\
\text { Hardness } \\
\text { (Hv) }\end{array}$ & S.D. \\
\hline $\begin{array}{l}\text { Original } \\
\text { sample }\end{array}$ & 248.81 & 5.13 & 17.69 & 0.71 & 1215.40 & 1.20 \\
\hline pH 2.0 & 218.81 & 5.03 & 14.09 & 0.65 & 1213.21 & 2.06 \\
\hline pH 3.5 & 219.97 & 4.83 & 14.72 & 0.48 & 1214.58 & 2.73 \\
\hline pH 5.0 & 220.16 & 4.92 & 14.92 & 0.65 & 1215.31 & 3.29 \\
\hline pH 7.0 & 221.15 & 4.37 & 15.12 & 0.74 & 1213.09 & 2.78 \\
\hline pH 8.5 & 220.36 & 4.16 & 14.99 & 0.72 & 1215.39 & 2.64 \\
\hline pH 9.5 & 219.80 & 4.26 & 14.82 & 0.66 & 1215.24 & 2.99 \\
\hline
\end{tabular}

(Section 3) 


\section{Table 3 - Total Mass Loss of the specimens after $20 \mathrm{~h}$ exposure}

Experimental Conditions

pH 2.0 with erosive particles

pH 3.5 with erosive particles

pH 5.0 with erosive particles

pH 7.0 with erosive particles

pH 8.5 with erosive particles

pH 9.5 with erosive particles

pH 2.0 without erosive particles

pH 9.5 without erosive particles

Immersion in solution of pH 2.0 (No particles) $\quad 0.004$

Immerssion in solution of pH 9.5 (No particles) $\quad 0.004$
Total Mass Loss (kg $\mathbf{m}^{-2}$ )

0.587

0.566

0.388

0.286

0.311

0.337

0.007

0.006

(Section 3) 


\section{Captions for Figures}

Fig. 1 - Schematic diagram of apparatus used for wear tests (a) Impinging jet particle slurry erosion apparatus (b) Ejector (Section 2.1)

Fig. 2 - XRD analysis of the original Y-TZP specimen

Fig. 3 - XRD analysis of the specimens eroded at $\mathrm{pH} 7.0$ at $90^{\circ}$ (Section 3 )

Fig. 4 - XRD analysis of the specimens eroded at $\mathrm{pH} 9.5$ at $90^{\circ}$ (Section 3)

Fig. 5 - XRD analysis of the specimens eroded at $\mathrm{pH} 2.0$ at $90^{\circ}$ (Section 3)

Fig. 6 - SEM of Y-TZP specimen before impact by solid particles at $90^{\circ}$ at $\mathrm{pH} 7$ after 20 h (Section 3)

Fig. 7 - SEM of Y-TZP specimen after impact by solid particles at $90^{\circ}$ at $\mathrm{pH} 7$ after $20 \mathrm{~h}$ (Section 3)

Fig. 8 - SEM of a cross-section of Y-TZP specimen after erosion-corrosion test (Section 3) at $\mathrm{pH} 7$ after $20 \mathrm{~h}$ (Section 3)

Fig. 9 - SEM of Y-TZP specimen after impact by solid particles at $15^{\circ}$ at $\mathrm{pH} 7$ after $20 \mathrm{~h}$ (Section 3)

Fig. 10 - SEM of Y-TZP specimen after impact by solid particles at $30^{\circ}$ at $\mathrm{pH} 7$ after 20 h (Section 3)

Fig. 11 - SEM of Y-TZP specimen after impact by solid particles at $45^{\circ}$ at $\mathrm{pH} 7$ after 20 h (Section 3)

Fig. 12 - SEM of Y-TZP specimen after impact by solid particles at $60^{\circ}$ after $20 \mathrm{~h}$ (Section 3) 
Fig. 13 - Accumulated mass loss curves at $90^{\circ}\left(\mathrm{kg} \mathrm{m}^{-2}\right)$

Fig. 14 - Accumulated mass loss curves at $15^{\circ}\left(\mathrm{kg} \mathrm{m}^{-2}\right)$

Fig. 15 - Accumulated mass loss curves at $30^{\circ}\left(\mathrm{kg} \mathrm{m}^{-2}\right)$

Fig. 16 - Accumulated mass loss curves at $45^{\circ}\left(\mathrm{kg} \mathrm{m}^{-2}\right)$

Fig. 17 - Accumulated mass loss curves at $60^{\circ}\left(\mathrm{kg} \mathrm{m}^{-2}\right)$

Fig. 18 - Wear map at 90 (Green: Low, Orange: Medium, Red: High)

Fig. 19 - Wear rate map at $90^{\circ}$ (Green: Low, Orange: Medium, Red: High)

Fig. 20 - Wear rate map at $15^{\circ}$ (Green: Low, Orange: Medium, Red: High)

Fig. 21 - Wear rate map at 30 (Green: Low, Orange: Medium, Red: High)

Fig. 22 - Wear rate map at $45^{\circ}$ (Green: Low, Orange: Medium, Red: High)

Fig. 23 - Wear rate map at $60^{\circ}$ (Green: Low, Orange: Medium, Red: High)

Fig. 24 - Wear phase transformation map at 90 (Light Green: Tetragonal, Pink:

Tetragonal + Monoclinic) 


\section{Figures}

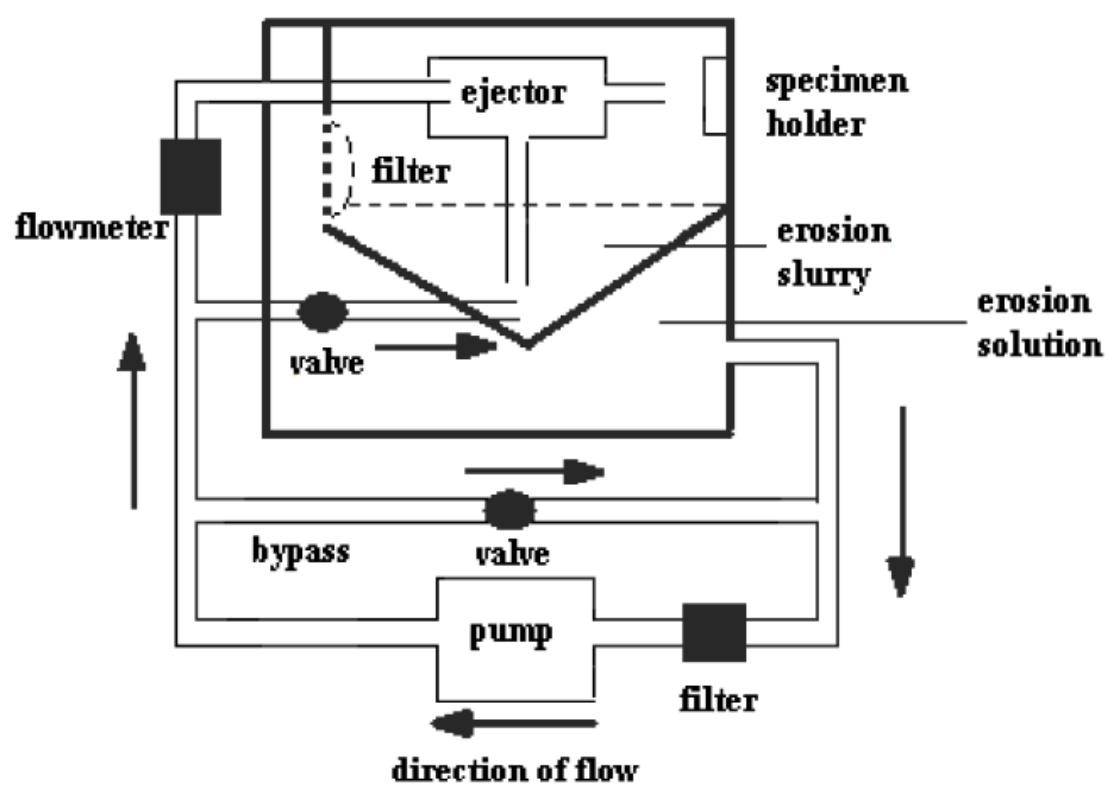

Fig. 1(a) Impinging jet particle slurry erosion apparatus

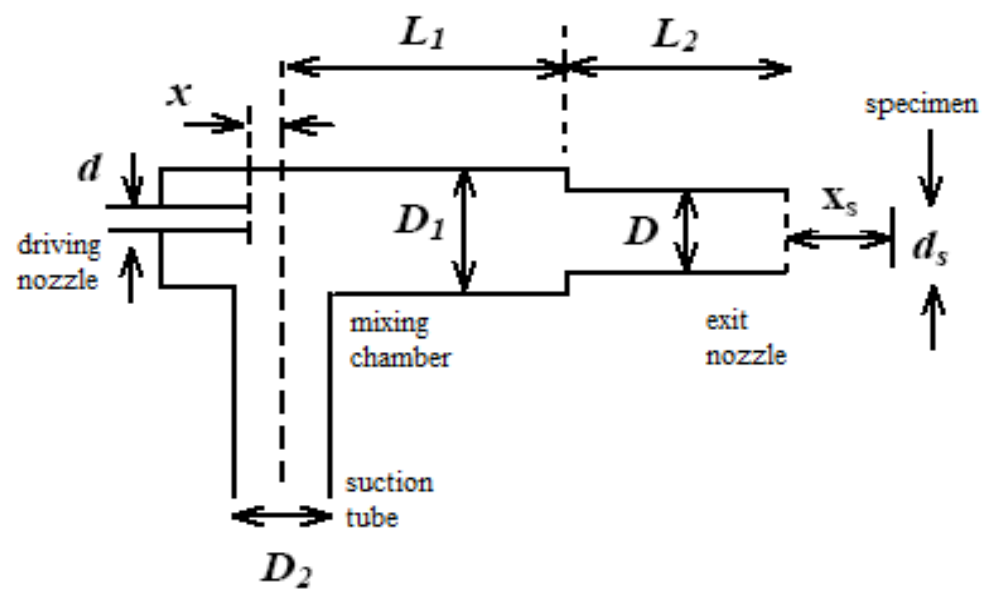

Fig.1(b) Ejector (Section 2.1)

Fig. 1 Schematic diagrams of apparatus for wear tests 


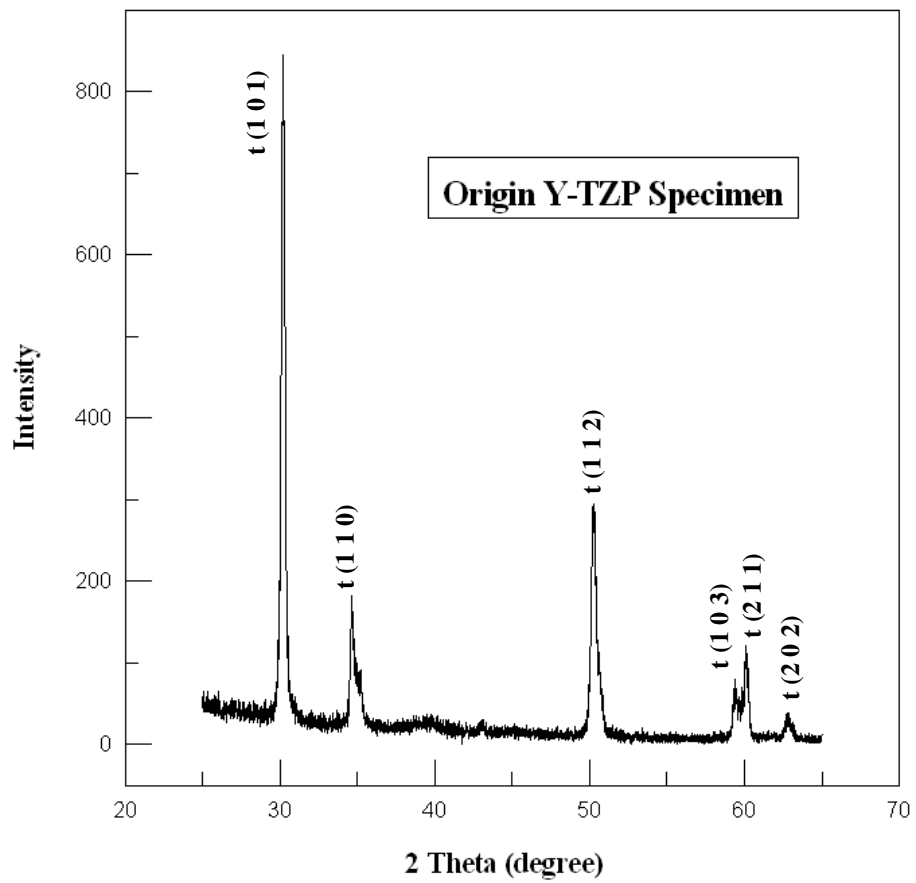

Fig. 2 - XRD analysis of the original Y-TZP specimen

(Section 3)

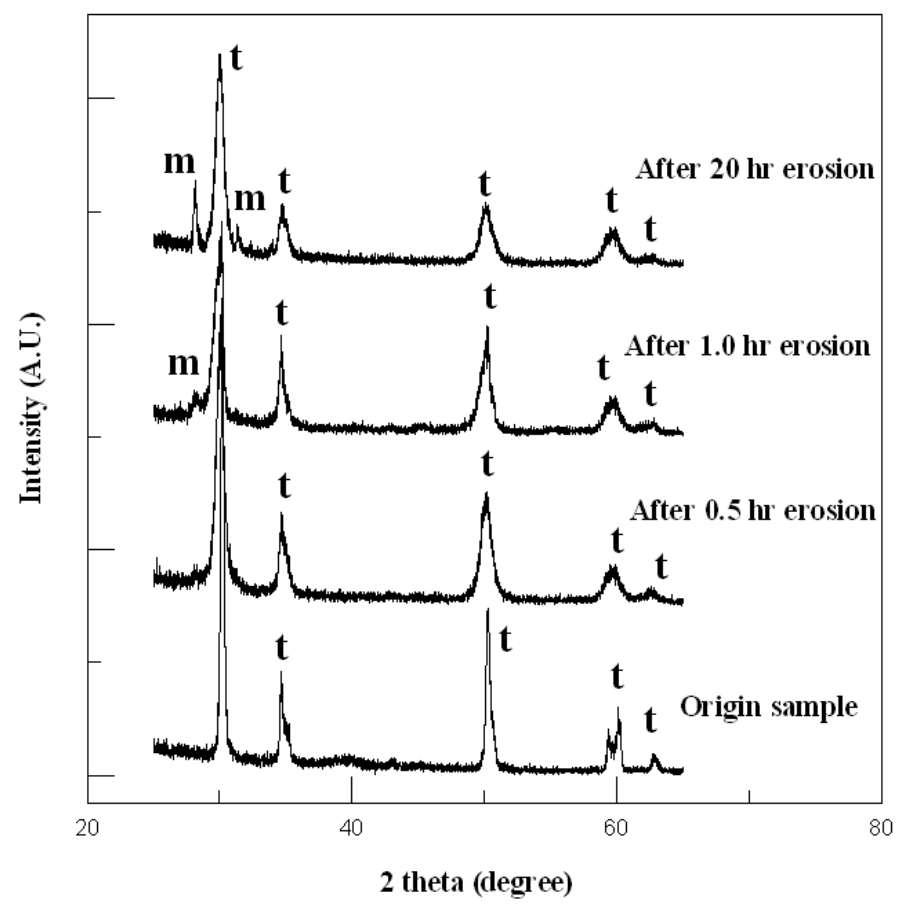

Fig. 3 - XRD analysis of the specimens eroded at $\mathrm{pH} 7.0$ at $90^{\circ}$ (Section 3 ) 


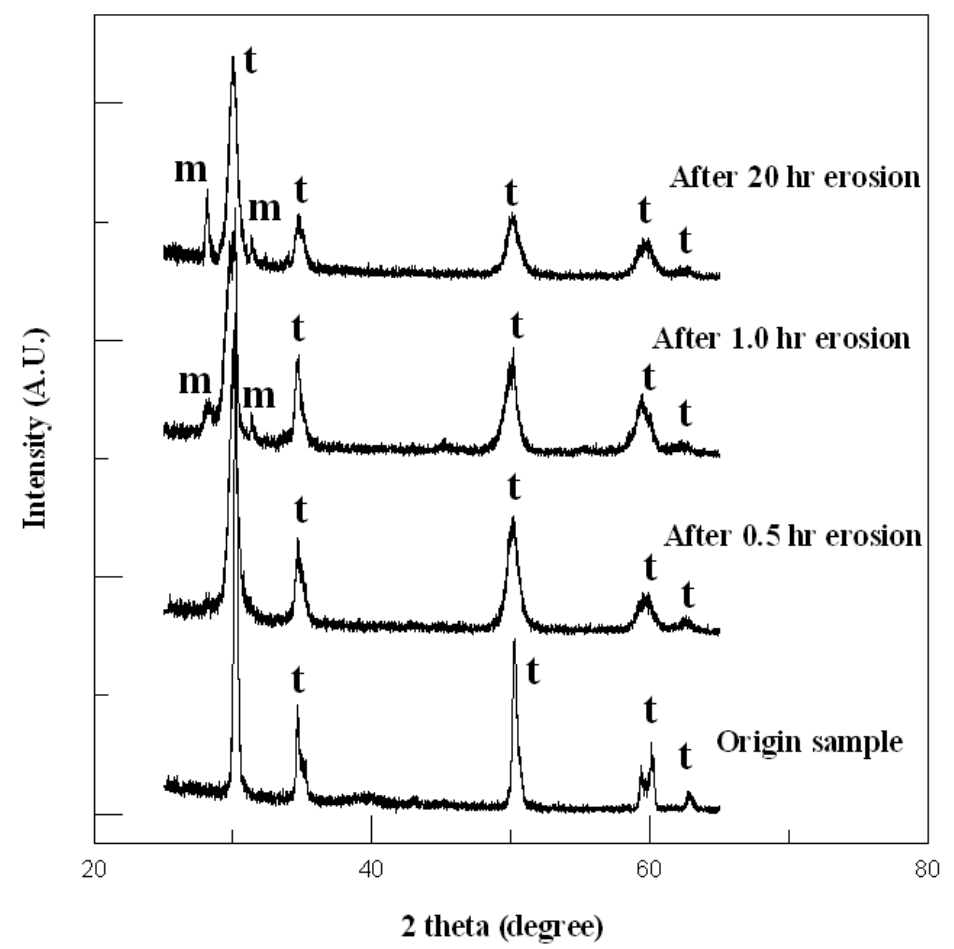

Fig. 4 - XRD analysis of the specimens eroded at $\mathrm{pH} 9.5$ at $90^{\circ}$ (Section 3)

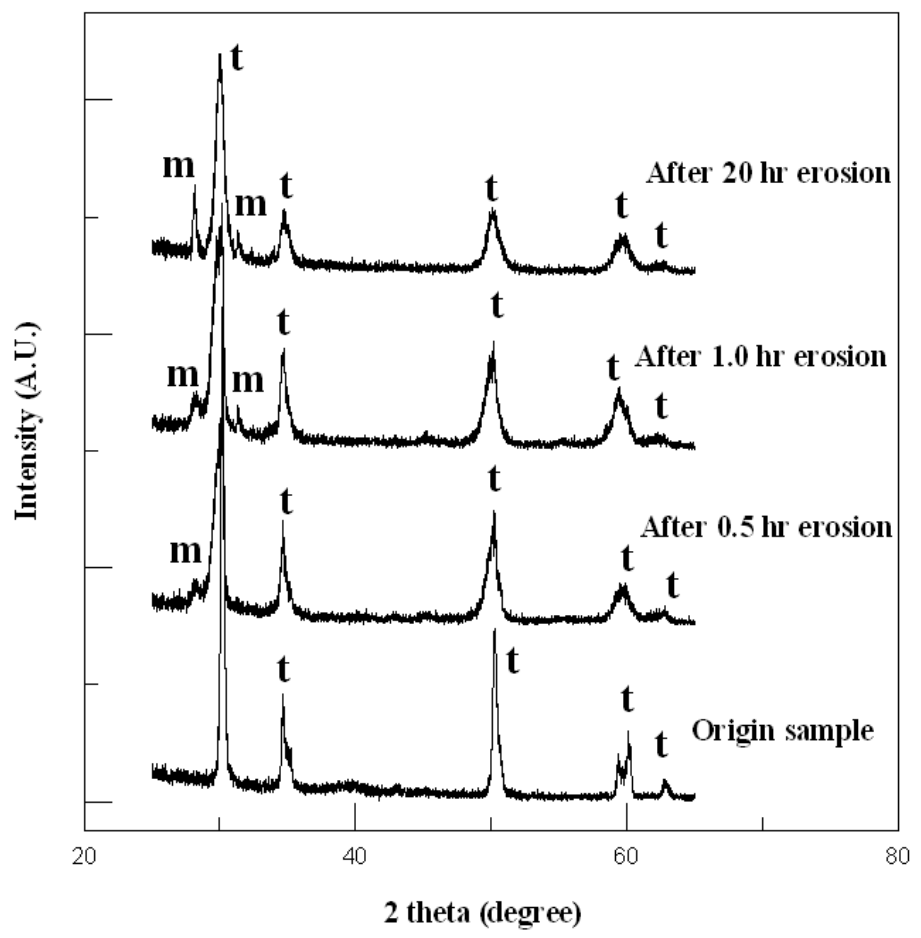

Fig. 5 - XRD analysis of the specimens at $\mathrm{pH}$ at $90^{\circ} 2.0 \quad$ (Section 3) 


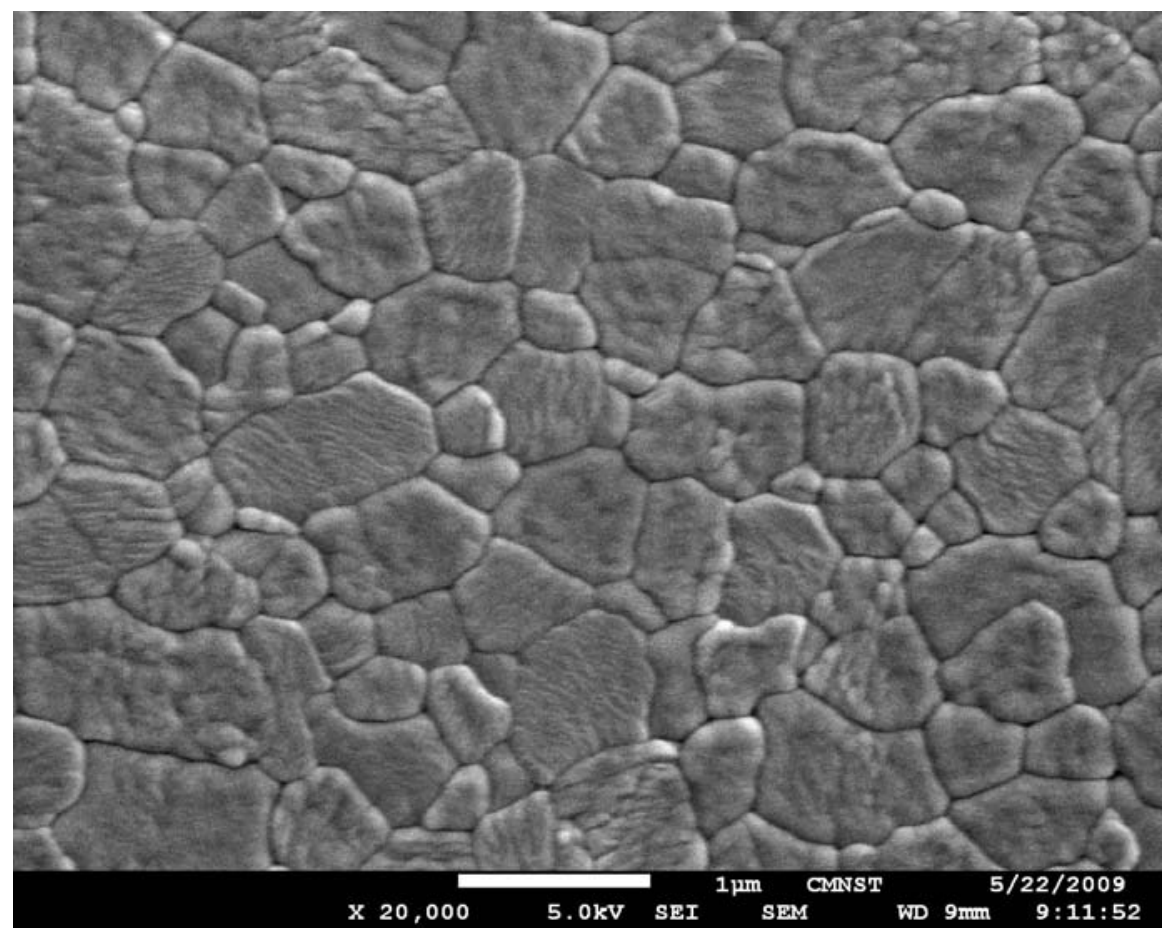

Fig. 6 - SEM of Y-TZP specimen before impact by solid particles at $90^{\circ}$ at $\mathrm{pH} 7$ after 20 h (Section 3)

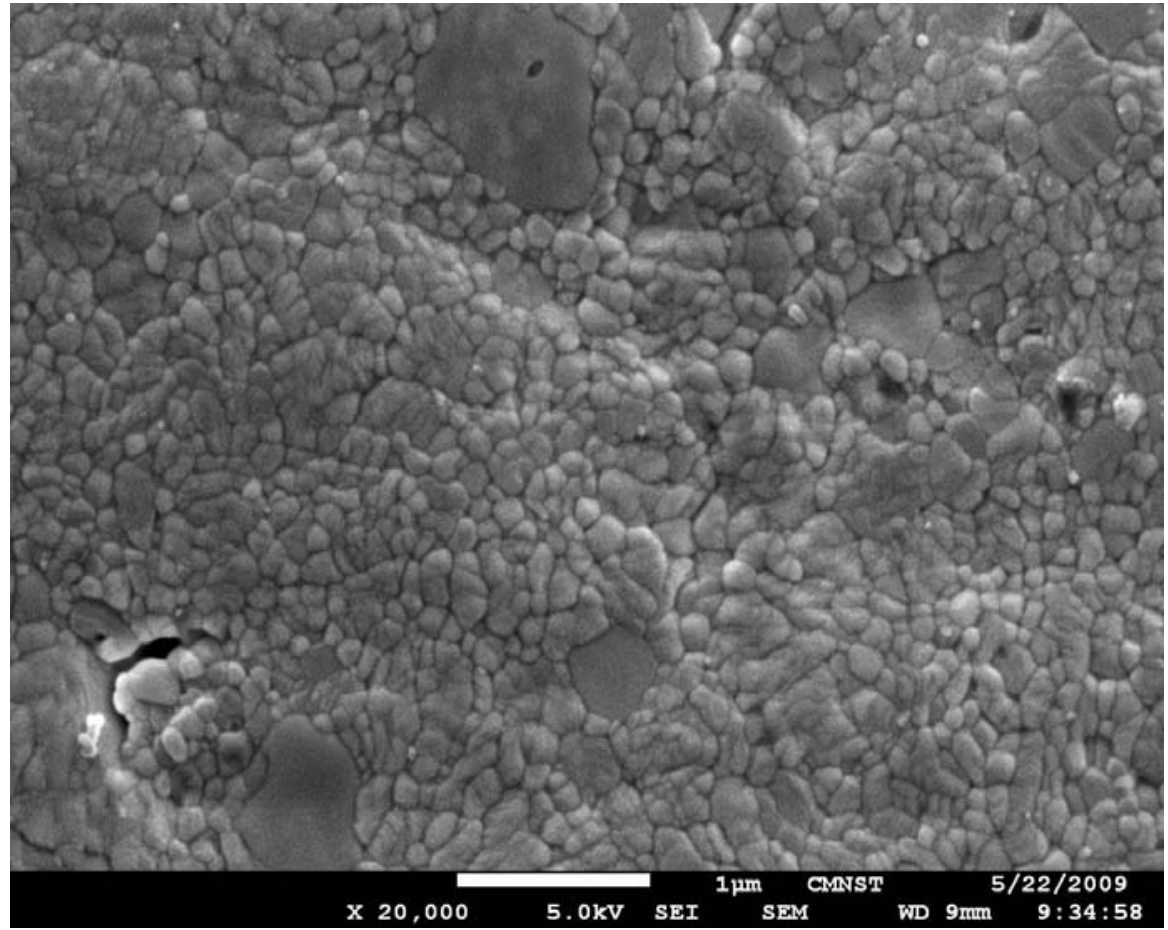

Fig. 7 - SEM of Y-TZP specimen after impact by solid particles at $90^{\circ}$ at $\mathrm{pH} 7$ after $20 \mathrm{~h}$ (Section 3) 


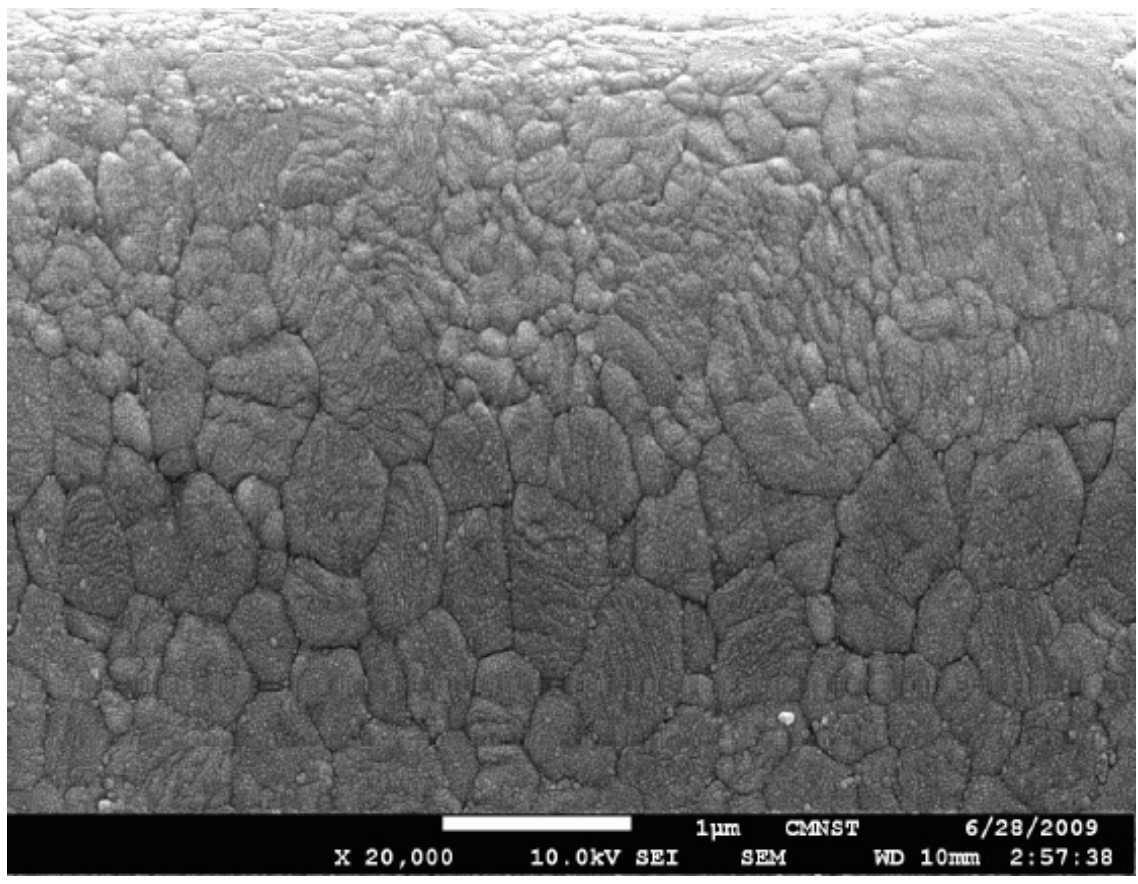

Fig. 8 - SEM observation of a cross-section of Y-TZP specimen after impact by solid particles at $60^{\circ}$ after $20 \mathrm{~h}($ Section 3$)$ at $\mathrm{pH} 7$.

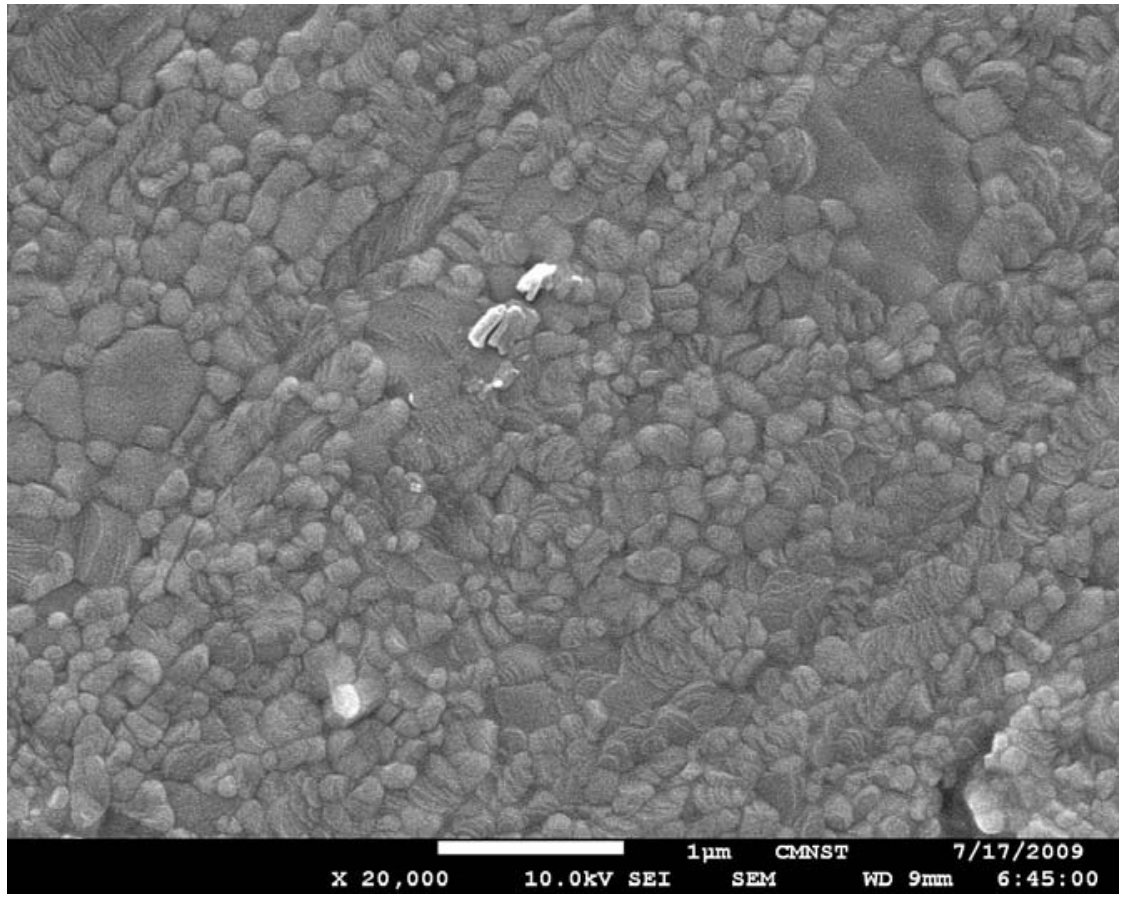

Fig. 9 - SEM observation of Y-TZP after impact by solid particles at $15^{\circ}$ after $20 \mathrm{~h}$ (Section 3) 


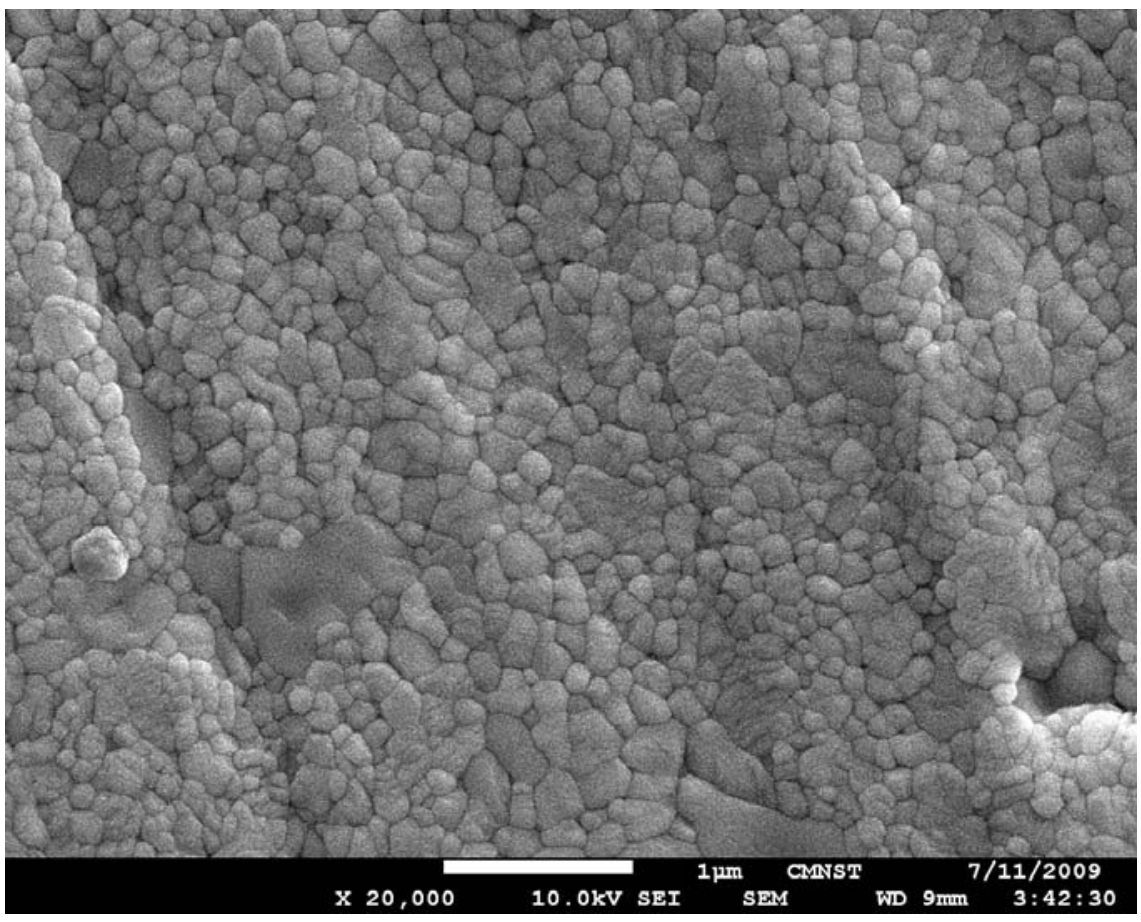

Fig. 10 - SEM observation of Y-TZP specimen after $30^{\circ}$ erosion test

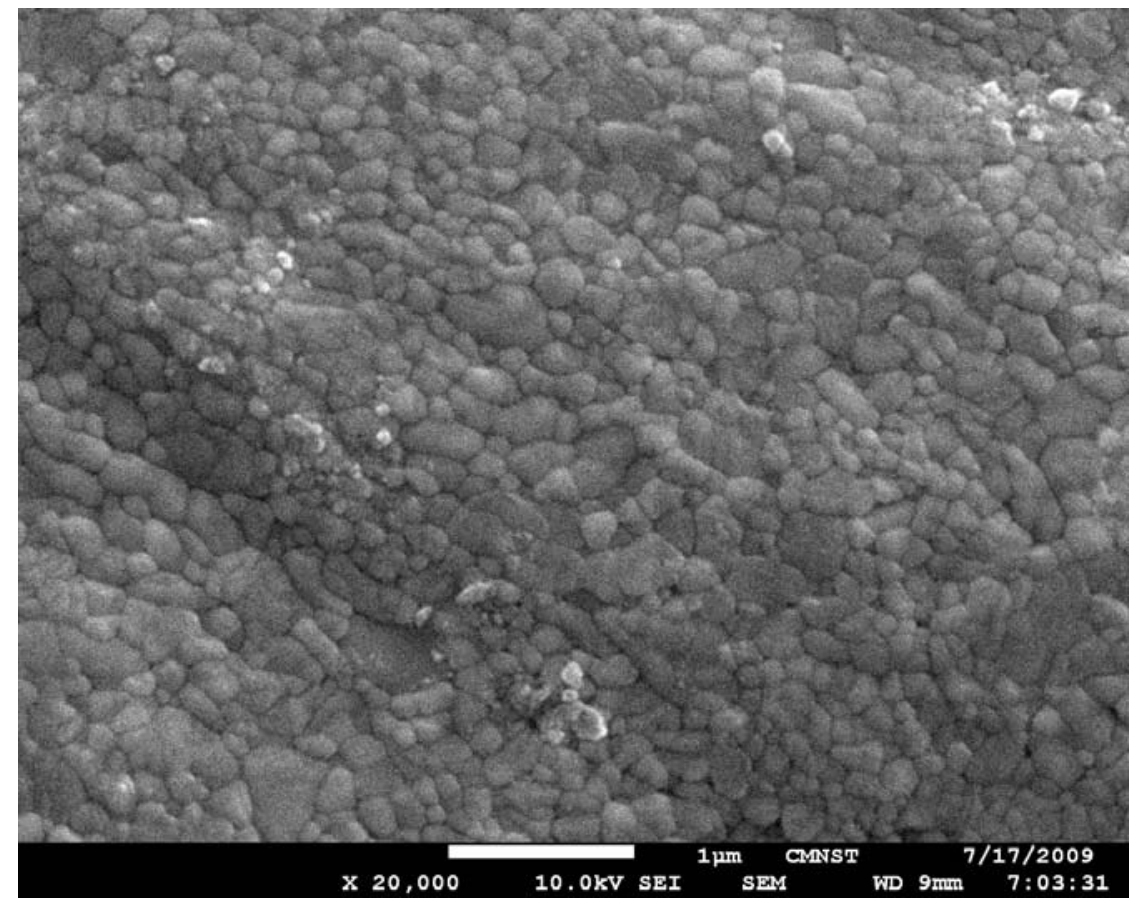

Fig. 11 - SEM observation of Y-TZP specimen after impact by solid particles at $45^{\circ}$ after 20h (Section 3) 


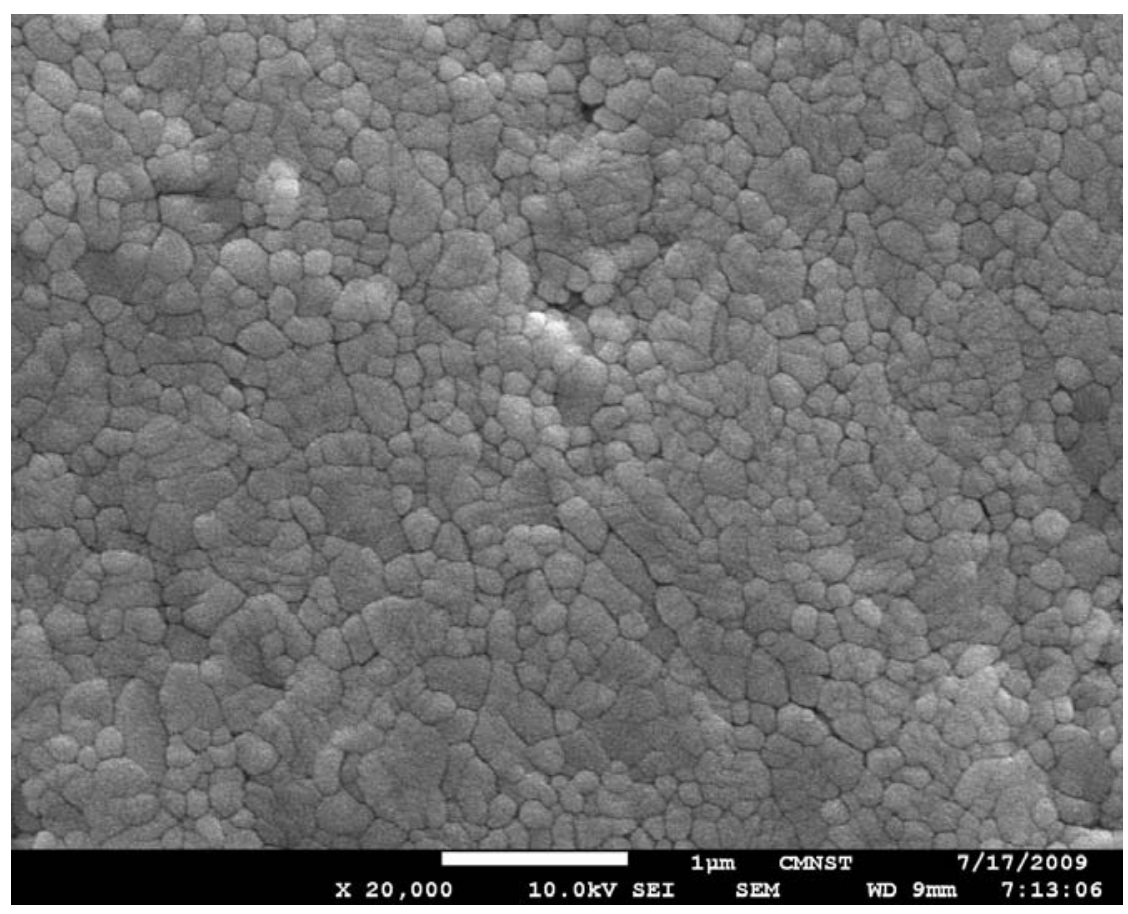

Fig. 12 - SEM observation of Y-TZP specimen after impact by solid particles at $60^{\circ}$ after 20h (Section 3) 


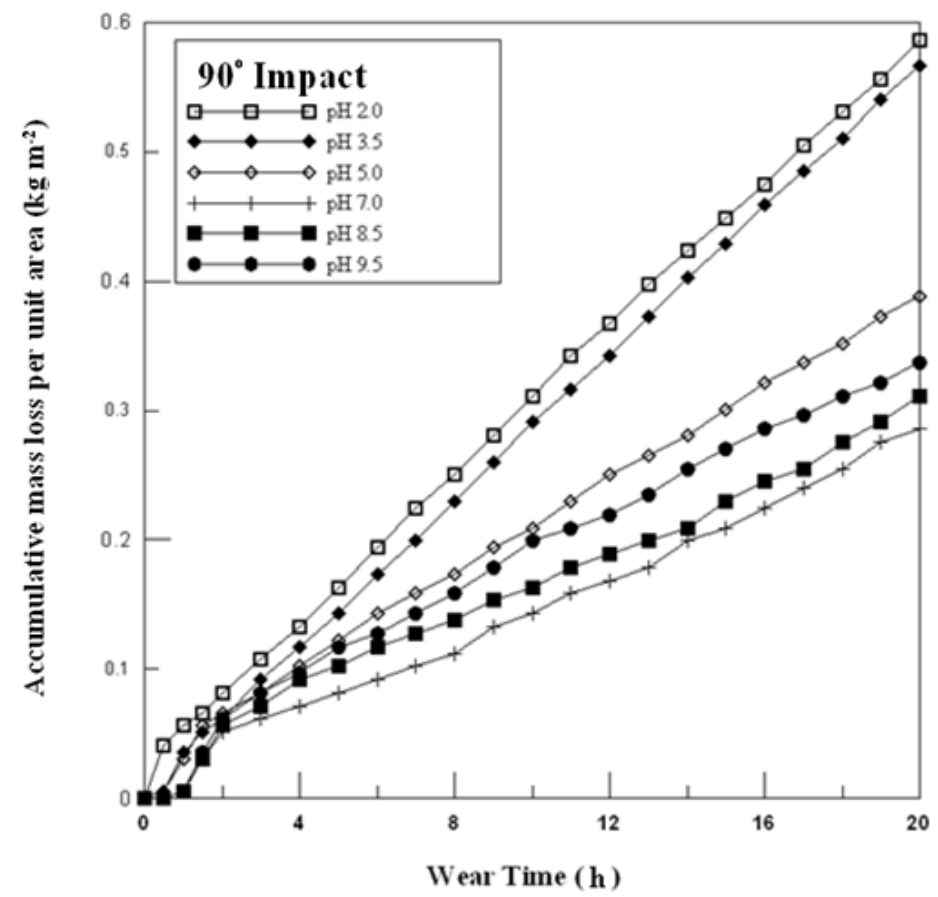

Fig. 13 - Accumulated mass loss curves at $90^{\circ}\left(\mathrm{kg} \mathrm{m}^{-2}\right)$

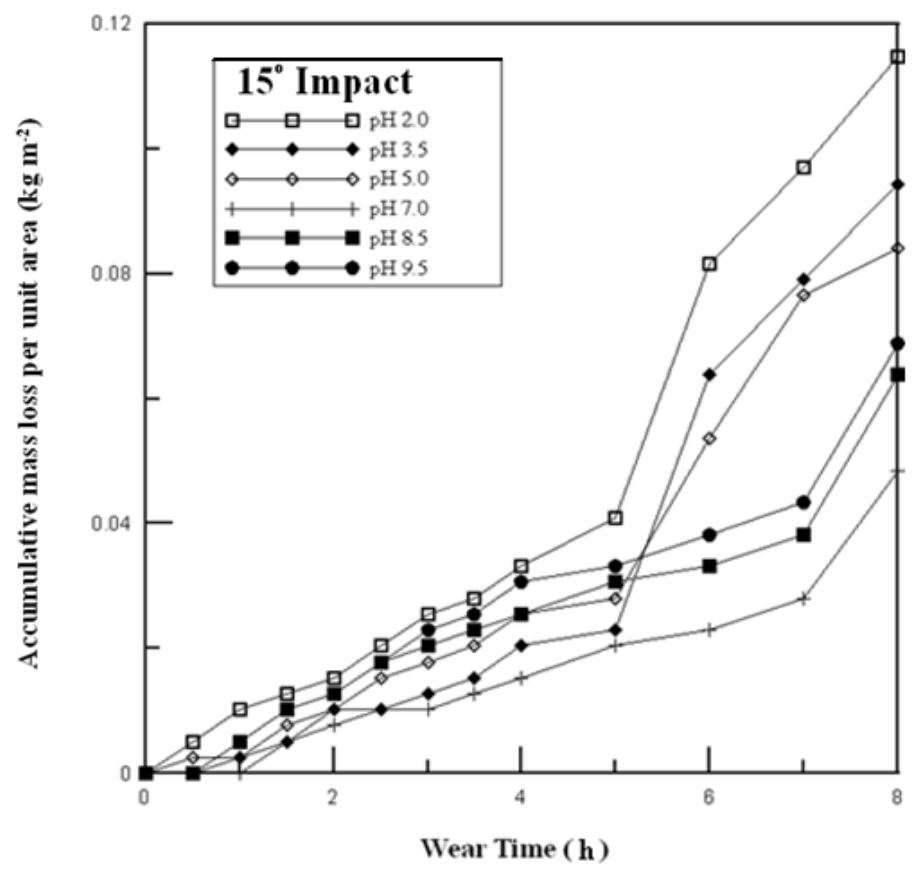

Fig. 14 - Accumulated mass loss curve at $15^{\circ}\left(\mathrm{kg} \mathrm{m}^{-2}\right)$ 


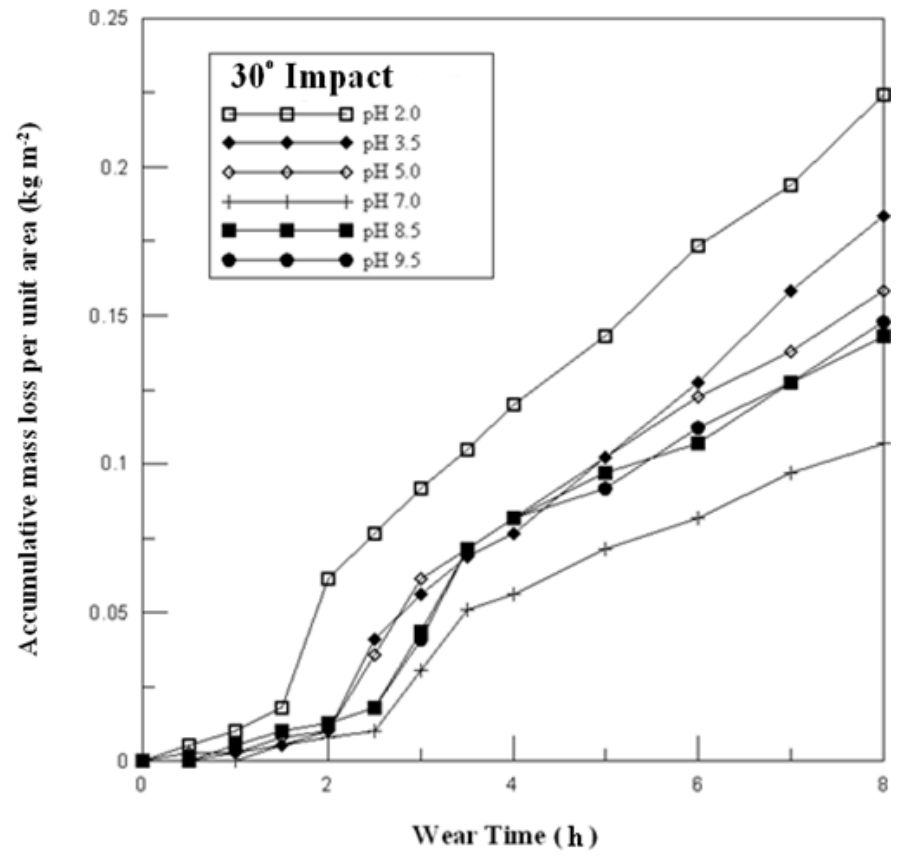

Fig. 15 - Accumulated mass loss curve at $30^{\circ}\left(\mathrm{kg} \mathrm{m}^{-2}\right)$

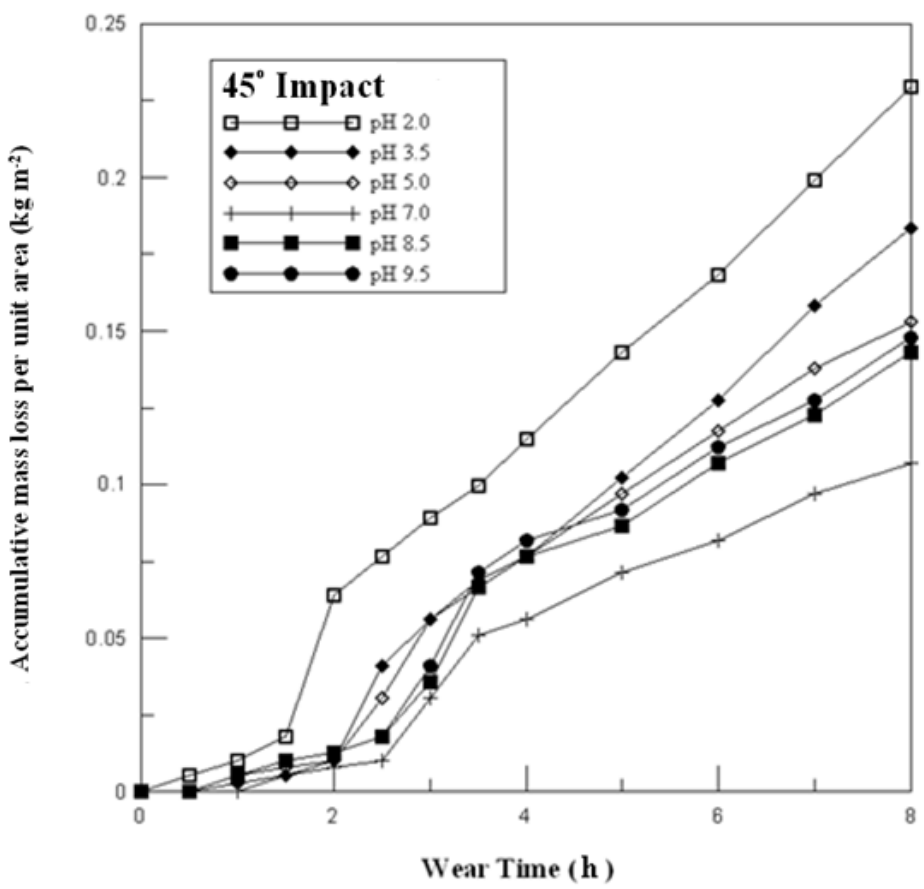

Fig. 16 - Accumulated mass loss curve at $45^{\circ}\left(\mathrm{kg} \mathrm{m}^{-2}\right)$ 


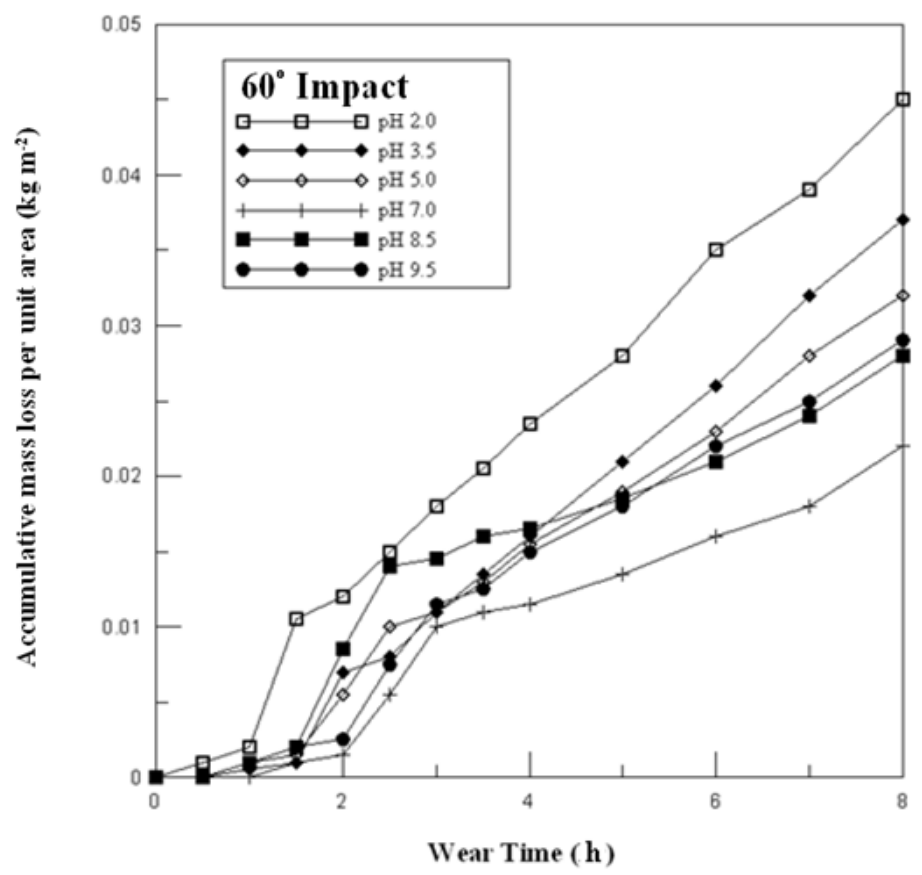

Fig. 17 - Accumulated mass loss curve at $60^{\circ}\left(\mathrm{kg} \mathrm{m}^{-2}\right)$

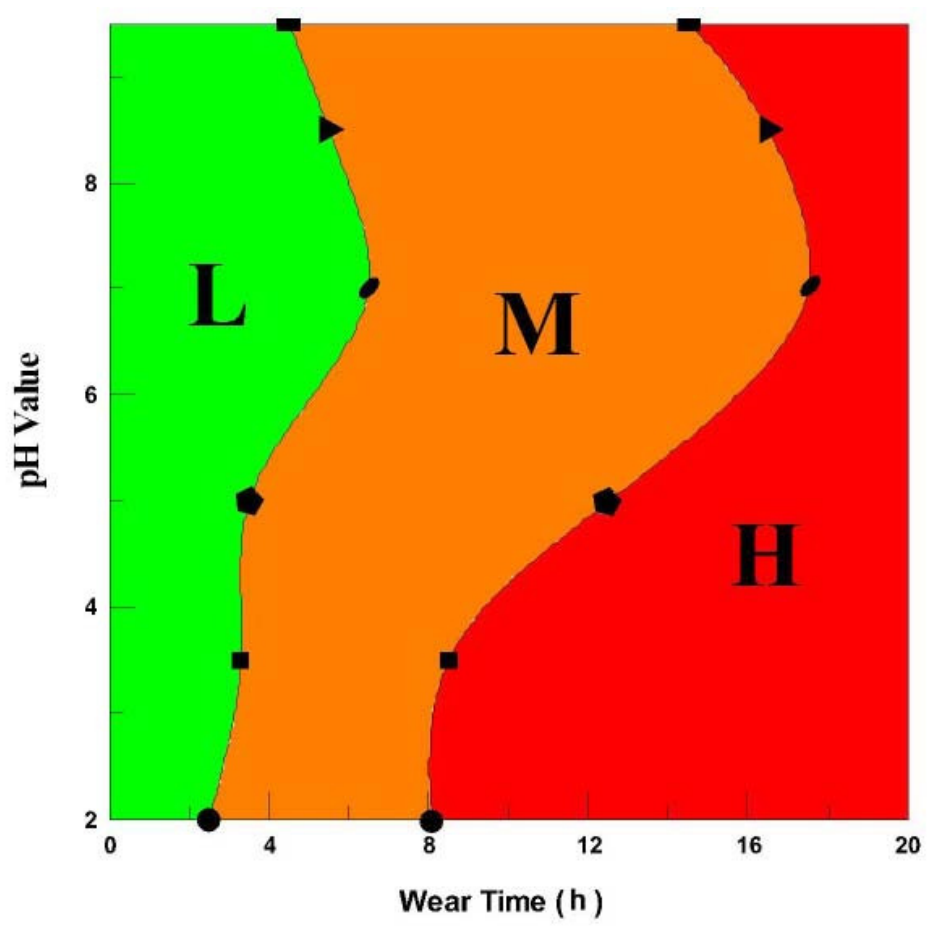

Fig. 18 - Wear map at $90^{\circ}$ (Green: Low, Orange: Medium, Red: High) 


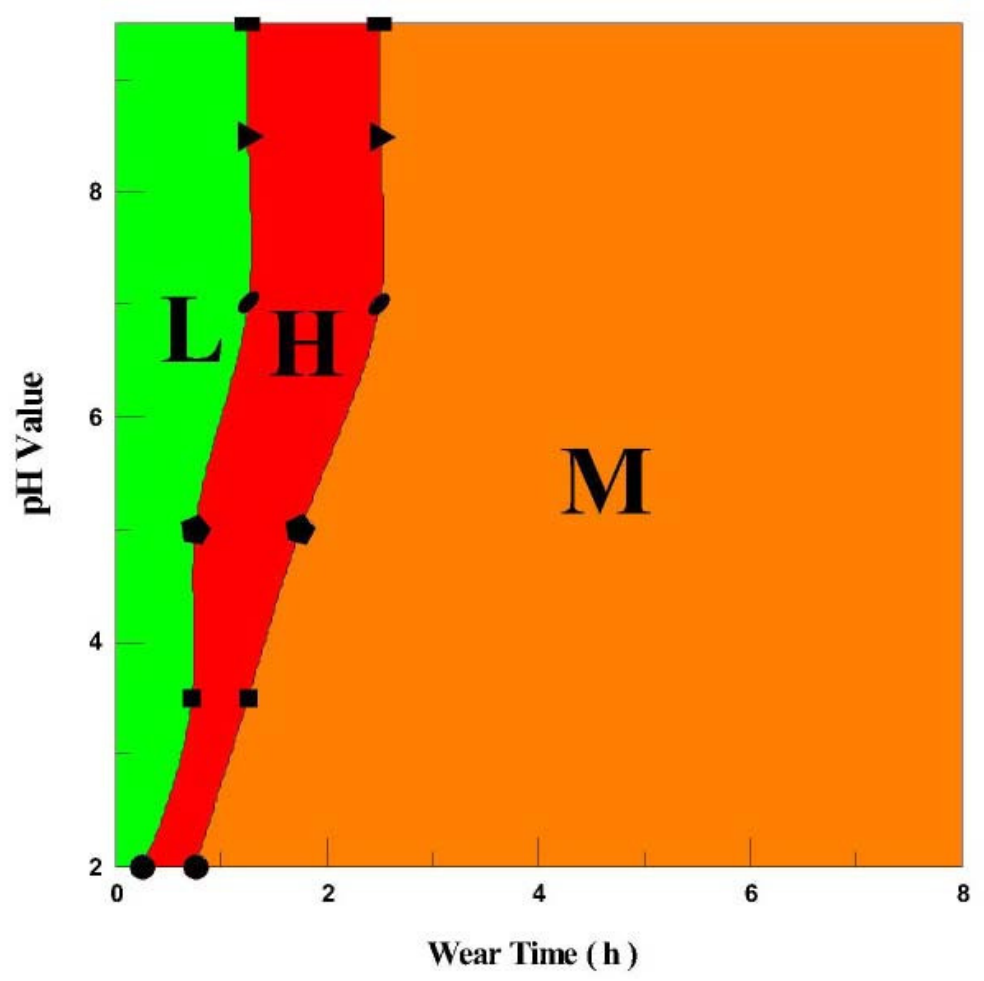

Fig. 19 - Wear rate map at 90 (Green: Low, Orange: Medium, Red: High)

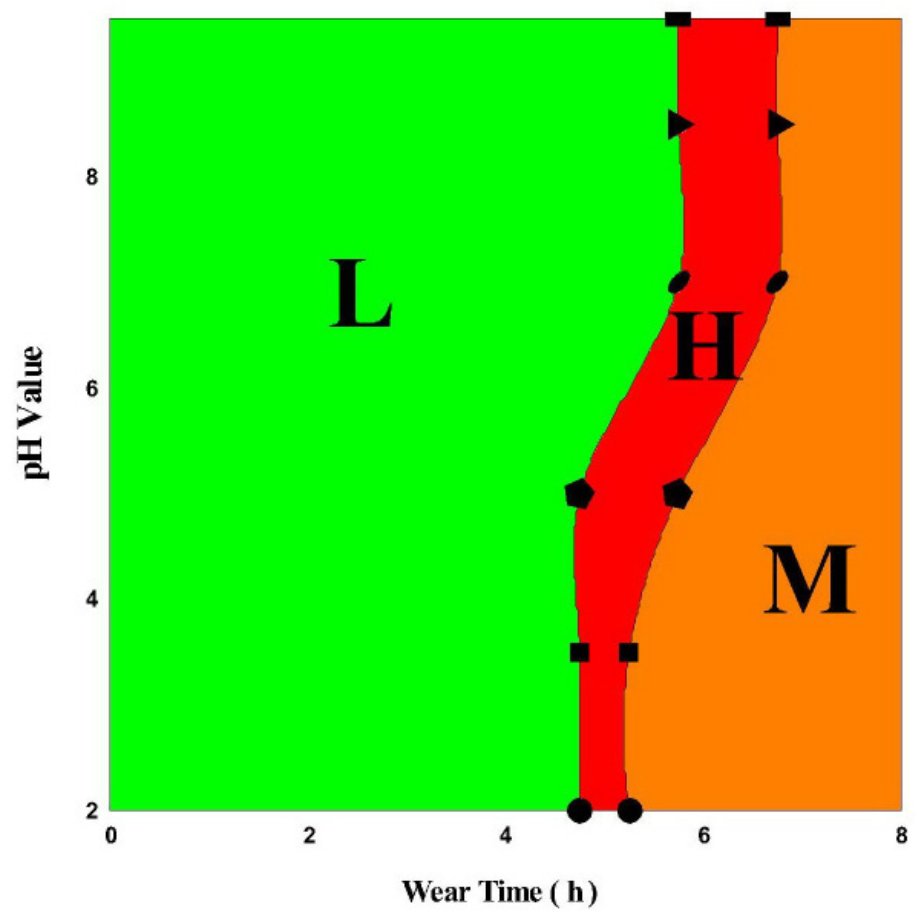

Fig. 20 - Wear rate map at $15^{\circ}$ (Green: Low, Orange: Medium, Red: High) 


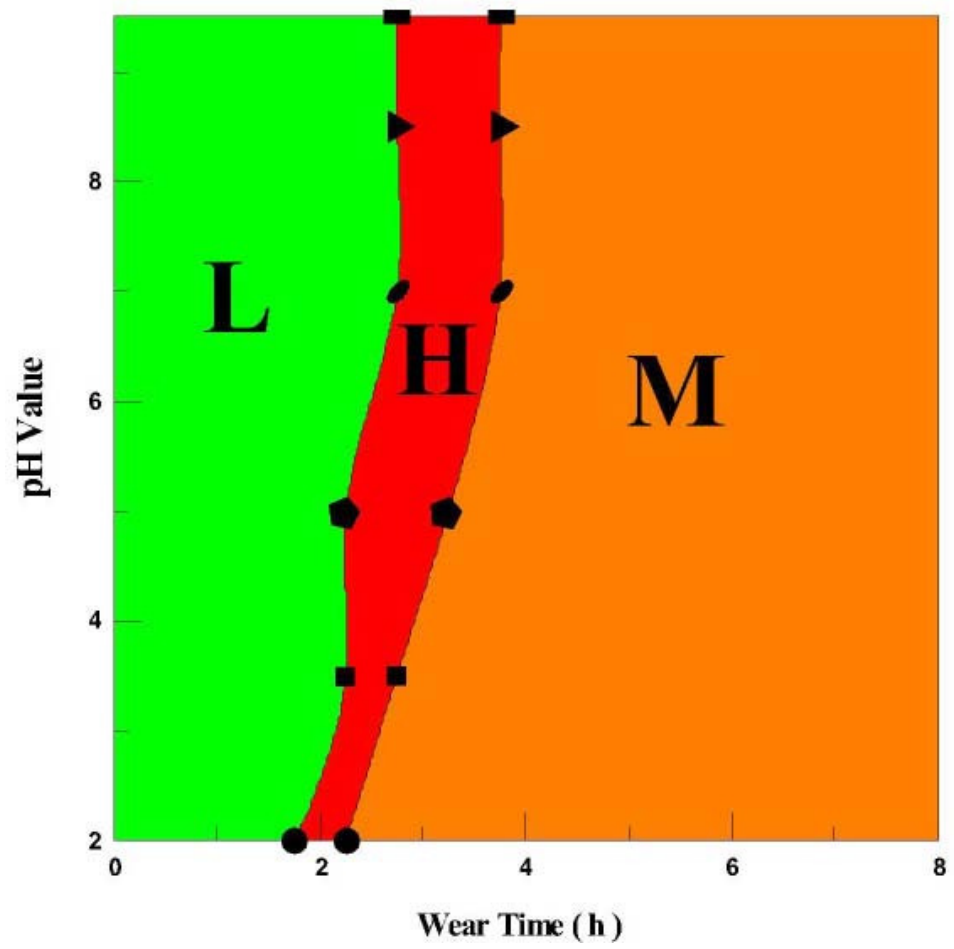

Fig. 21 - Wear rate map at 30 (Green: Low, Orange: Medium, Red: High)

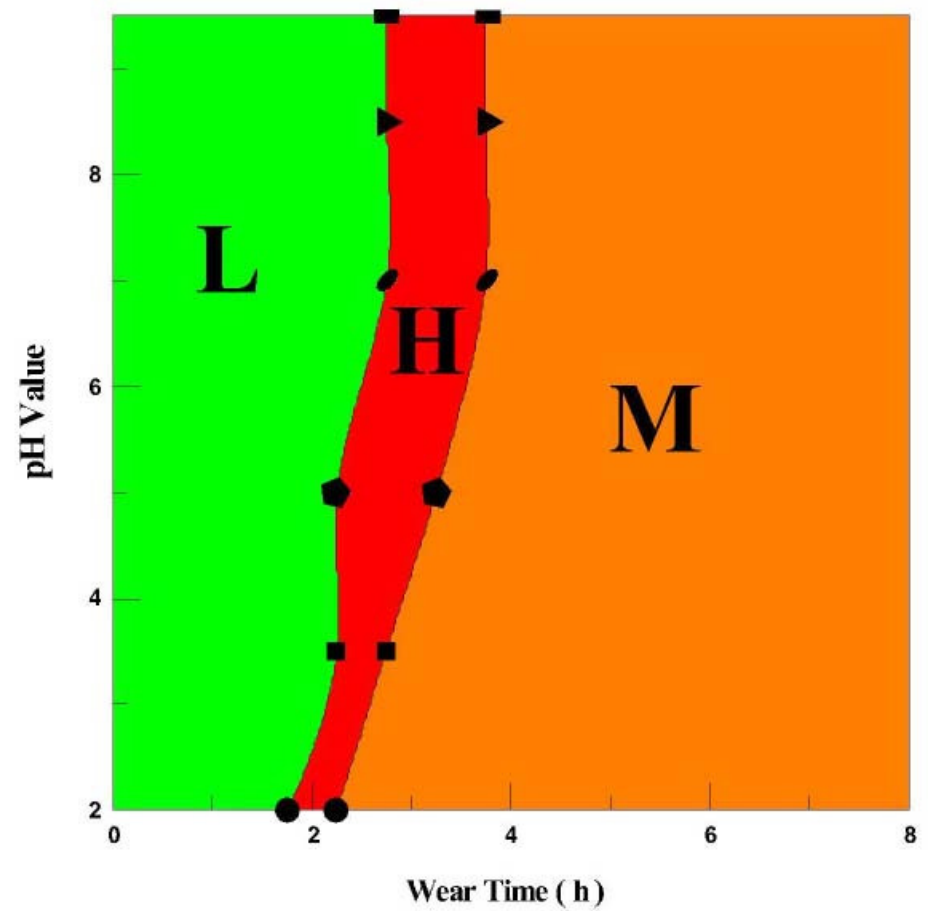

Fig. 22 - Wear rate map at $45^{\circ}$ (Green: Low, Orange: Medium, Red: High) 


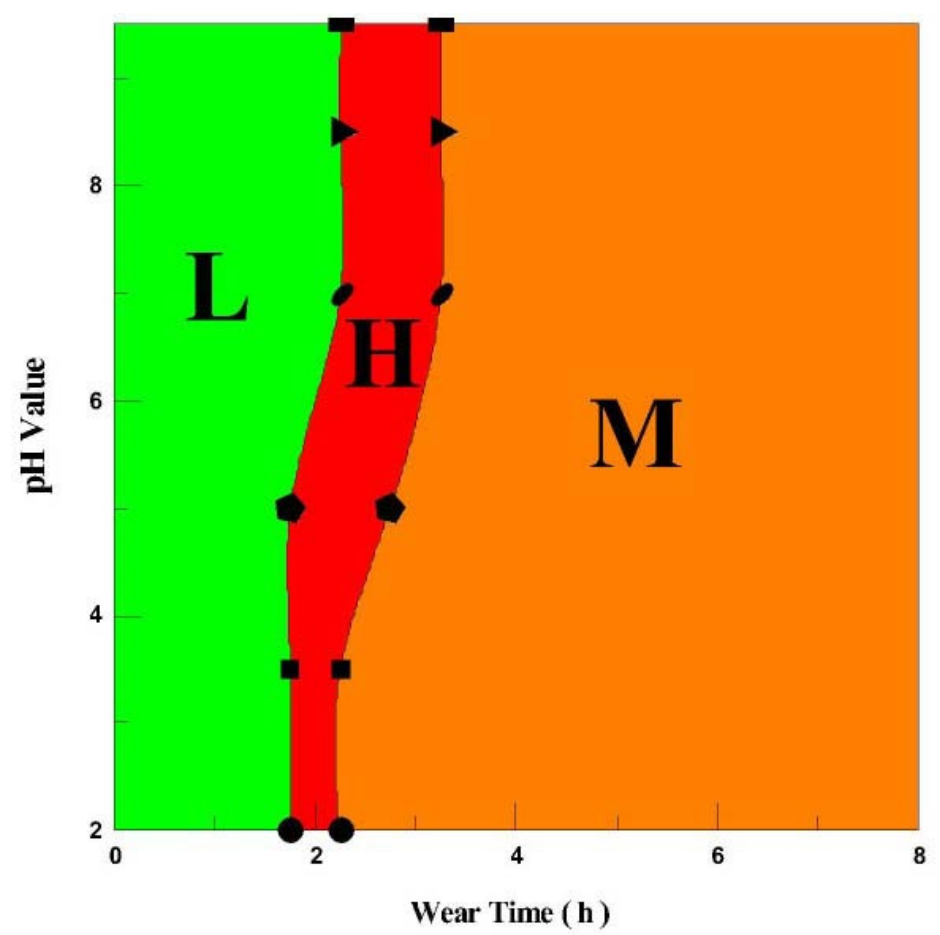

Fig. 23 - Wear rate map at $60^{\circ}$ (Green: Low, Orange: Medium, Red: High)

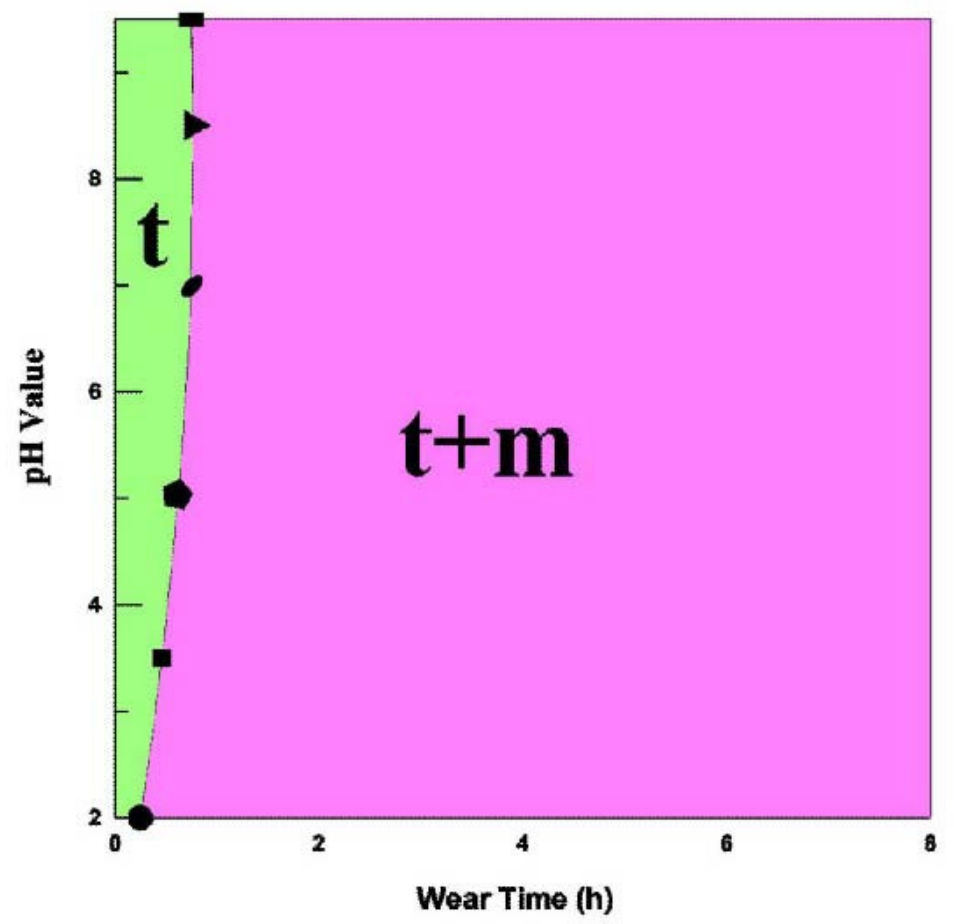

Fig. 24 - Wear phase transformation map at $90^{\circ}$ (Light Green: Tetragonal, Pink: 
Tetragonal + Monoclinic) 